\title{
Selection and characterization of llama single domain antibodies against $\mathbf{N}$-terminal huntingtin
}

\author{
Menno H. Schut • Barry A. Pepers · Rinse Klooster • \\ Silvère M. van der Maarel • Mohamed el Khatabi · Theo Verrips • \\ Johan T. den Dunnen · Gert-Jan B. van Ommen · Willeke M. C. van Roon-Mom
}

Received: 7 July 2014/ Accepted: 24 September 2014/Published online: 8 October 2014

(C) The Author(s) 2014. This article is published with open access at Springerlink.com

\begin{abstract}
Huntington disease is caused by expansion of a CAG repeat in the huntingtin gene that is translated into an elongated polyglutamine stretch within the $\mathrm{N}$-terminal domain of the huntingtin protein. The mutation is thought to introduce a gain-of-toxic function in the mutant huntingtin protein, and blocking this toxicity by antibody binding could alleviate Huntington disease pathology. Llama single domain antibodies ( $\mathrm{VHH}$ ) directed against mutant huntingtin are interesting candidates as therapeutic agents or research tools in Huntington disease because of their small size, high thermostability, low cost of production, possibility of intracellular expression, and potency of blood-brain barrier passage. We have selected VHH from llama phage display libraries that specifically target the $\mathrm{N}$-terminal domain of the huntingtin protein. Our VHH are capable of binding wild-type and mutant human huntingtin
\end{abstract}

Electronic supplementary material The online version of this article (doi:10.1007/s10072-014-1971-6) contains supplementary material, which is available to authorized users.

M. H. Schut · B. A. Pepers - S. M. van der Maarel ·

J. T. den Dunnen - G.-J. B. van Ommen .

W. M. C. van Roon-Mom ( $\square)$

Department of Human Genetics, Center for Human and Clinical

Genetics, Leiden University Medical Center, Albinusdreef 2,

2333 ZA Leiden, The Netherlands

e-mail: W.M.C.van_Roon@lumc.nl

R. Klooster

Merus BV, Padualaan 8, 3584 CH Utrecht, The Netherlands

M. el Khatabi - T. Verrips

QVQ BV, Padualaan 8, 3584 CH Utrecht, The Netherlands

J. T. den Dunnen

Leiden Genome Technology Center, Center for Human and

Clinical Genetics, Leiden University Medical Center,

Albinusdreef 2, 2333 ZA Leiden, The Netherlands under native and denatured conditions and can be used in Huntington disease studies as a novel antibody that is easy to produce and manipulate.

Keywords VHH - Huntington disease - PolyQ . $\mathrm{N}$-terminal huntingtin $\cdot$ Huntingtin

\section{Introduction}

Huntington disease (HD) is caused by expansion of a CAG repeat within the first exon of the huntingtin gene (4p16.3) [1]. This mutation results in an expanded polyglutamine repeat (polyQ) at the $\mathrm{N}$-terminus of the huntingtin protein (htt), causing HD pathology through a toxic gain-of-function mechanism [2]. Antibody binding could reduce toxicity of the mutant htt protein. Messer et al. showed that a single chain Fv antibody construct, selected against the first $17 \mathrm{~N}$-terminal htt amino acids was capable of reducing HD pathogenesis in various HD models [3, 4]. In our study we make use of llama single domain antibody fragments called VHH [5]. VHH contain four framework regions (FR1-4) for structural integrity and three variable complement determining regions (CDR1-3) that usually determine epitope binding. VHH have distinctive advantages compared with other antibody classes. VHH are thermostable, only $\sim 16 \mathrm{kD}$ in size and their single domain nature simplifies selection and production [6,7]. VHH have been used for diseases such as oculopharyngeal muscular dystrophy (OPMD), which shares characteristics with HD. OPMD is caused by expansion of a triplet repeat in the $P A B P N 1$ gene that encodes for a polyalanine repeat at the $\mathrm{N}$-terminus of the polyA binding nuclear 1-protein (PABN1). VHH binding to an $\alpha$-helical domain of mutant PABN1 prevented aggregation [8], and alleviated OPMD 
Fig. 1 VHH protein sequences. iVHH 1-4 were selected from an immunized llama phage display library. nVHH was selected from a non-immunized llama phage display library. Underscored amino acid position differs from iVHH1. Amino acid positions, framework (FR, thin line) and complementary determining regions (CDR, thick line) are according to Kabat [25]. - , deletion

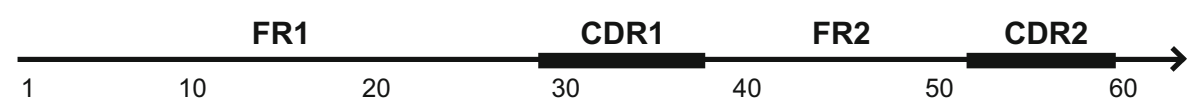

iVHH1-MAEVQLVESGGGLVQPGGS LRLSCAASGFALDYYA IGWFRQAPGKEREGVSCISATDGSTYYA iVHH2-MAEVQLVESGGGLVQPGGS LRLSCAAS GFS LDYYAIGWFRQAPGKEREGVSCISATDGSTYYA iVHH3-MAEVQLVESGGGLVQPGGS LRLSCAASGFTLDYYA IGWFRQAPGKEREGVSCISASDGSTYYA iVHH4-MAEVQLVESGGGLVQPGGS LRLSCAASGFTLDYYA IGWERQAPGKEREGVSCISSSDGSTYYA nVHH-MAQVQLQESGGGLVQAGGS LRLSCAASGRTFS SLYMGWFRQAPGKEREFVAS I SWS-GNTYYK

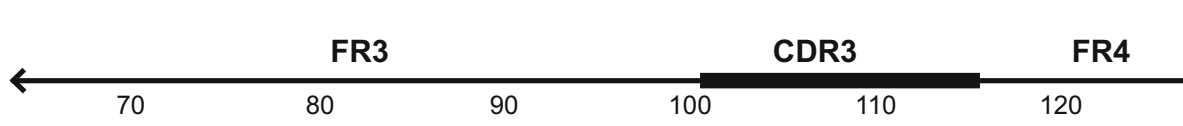

iVHH1-DSVKGRFT ISRDNAKNTVYLQMNS LKPEDTAVYYCATVRAPYSDYCNGYYDYWGQGTQVTVSS iVHH2-DSVKGRFT ISRDNAKNTVYLQMNS LKPEDTAVYYCATVRAPYS DYCNGYYDYWGQGTQVTVSS iVHH3-DSVKGRFT I SRDNAKNTVYLQMNSLKPEDTAVYYCATVRAPYS DYCNGYYDYWGQGTQVTVSS iVHH4-DSVKGRFT I SRDNAKNTVYLQMNSLKPEDTAVYYCATVRAPYS DYCNGYYDYWGQGTQVTVSS nVHH-DSLKGRVTISRDNAKNTVYLQMNSLKPEDTAVYYCA---GRRYPLVTGPYDIWGQGTQVTVSS pathology in a drosophilia model [9]. In the current study, we have selected VHH against the N-terminal domain of htt from llama phage display libraries. We show that high resolution melting curve analysis (HRMCA) [10] can successfully identify identical clones prior to sequencing. Our VHH can bind both endogenous and purified human wild-type and mutant htt at an epitope located between amino acids 49-148 and can co- immunoprecipitate htt from human HD brain lysates.

\section{Results}

Selection of VHH against N-terminal huntingtin

The phage-VHH (P-VHH) display library originated from llamas pre-immunized with an Escherichia coli produced $\mathrm{N}$-terminal htt protein fragment consisting of the first 548 amino acids with 46 polyQs. We performed four different selections; each selection involved two rounds using either a wild-type or mutant $\mathrm{N}$-terminal htt fragment. Enrichment of P-VHH after two rounds of selection was similar for direct coating or pre-capturing of N-terminal htt in the first round, with the optimal concentration being $5 \mu \mathrm{g} \mathrm{N}$-terminal htt (Online Resource 1a). P-VHH output numbers of up to $3 \times 10^{4}$ were obtained. Screening ELISA revealed that on average, $20 \%$ of selected clones bound htt (Online Resource 1b). Further screening by HRMCA (Online Resource 1c), followed by sequence analysis, revealed four
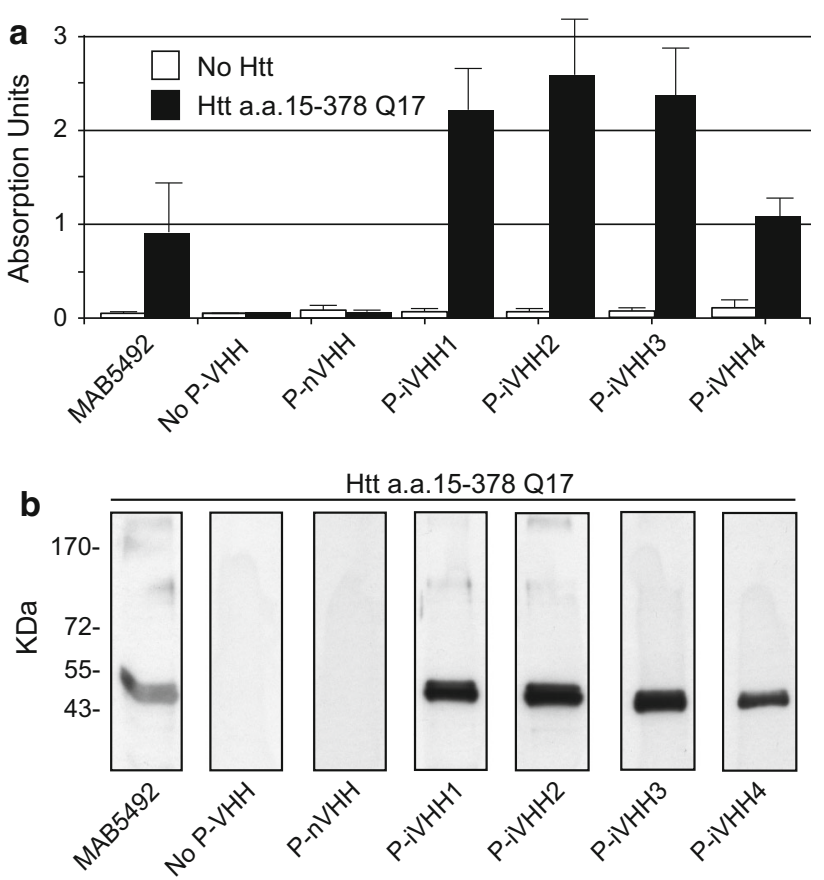

Fig. 2 VHH specificity for N-terminal htt. Assays were performed on a recombinant $\mathrm{N}$-terminal htt fragment consisting of amino acids 15-378 with a polyQ length of 17 (htt a.a. 15-378 Q17). Positive control: MAB5492. Negative control: No P-VHH or P-nVHH. a ELISA on wells with (black bars), or without (white bars) N-terminal htt. Bars represent mean ELISA signal from two independent ELISA assays with standard deviation. Each assay was performed in triplicate. ELISA absorption units are measured at $\lambda=490 \mathrm{~nm}$. b Western blotting on $\mathrm{N}$-terminal htt. Blots were performed twice. $k D a$ Molecular weight (kilodalton) 

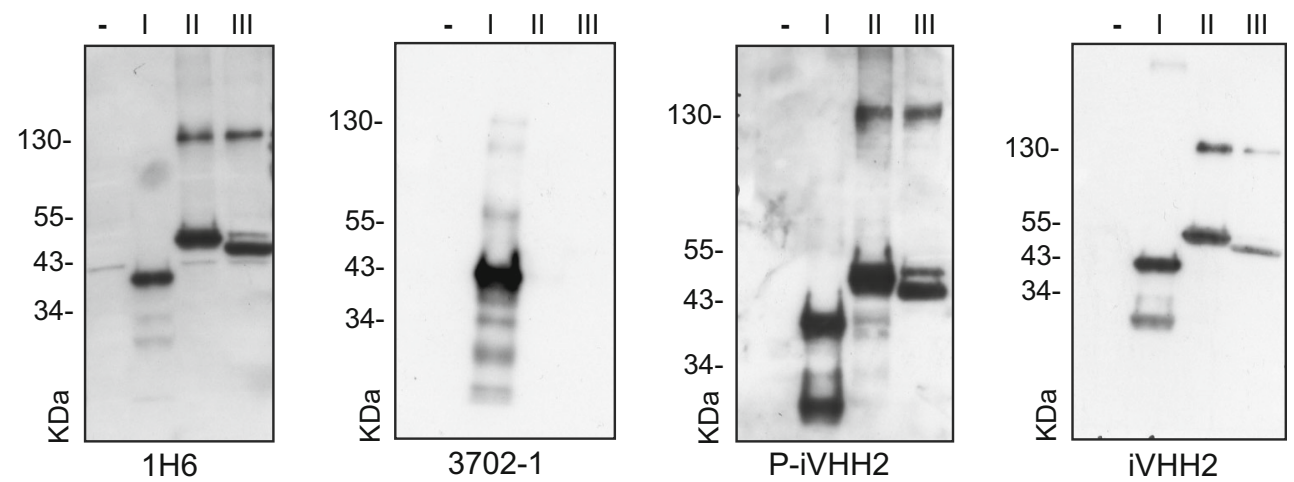

Fig. 3 VHH epitope determination. Western blots performed on no htt (-), htt a.a. 1-148 Q46 (I), htt a.a. 15-378 Q17 (II), and htt a.a. 49-415 (III). Primary antibody indicated below each blot. Blots were performed twice. $k D a$ Molecular weight (kilodalton)

htt specific VHH, (immune)VHH1-4. These differed by one, two, or three amino acid substitutions in the CDR1 and CDR2, while the CDR3 was identical (Fig. 1). The negative control VHH (n-VHH) was selected previously from a naive llama phage display library [10].

Specificity of monoclonal iVHH for N-terminal huntingtin

To investigate if the iVHH specifically bind htt, we performed ELISA and western blot analysis on a normal and mutant $\mathrm{N}$-terminal htt fragment using P-iVHH [11]. ELISA resulted in a positive signal for all P-iVHH (Fig. 2a, Online Resource 2a). P-iVHH1, 2 and 3 showed an equally strong ELISA signal, whereas P-iVHH4 gave a weaker signal. On western blot, all P-iVHH showed a band that matched the band obtained with the known htt antibody MAB5492 (Fig. 2b, Online Resource 2b). Western blotting results were in agreement with ELISA results.

\section{Epitope determination of iVHH on N-terminal} huntingtin

To determine the epitope of our iVHH, we performed western blotting using three different $\mathrm{N}$-terminal htt fragments. Each fragment had a partially overlapping sequence with the next fragment (Fig. 3). All fragments were recognized by the 1H6 antibody [12] while 3702-1, that binds htt at the N-terminal 13 amino acids (Online Resource 3a), only recognized fragment I. All P-iVHH recognized all fragments (Fig. 3, Online Resource 3b) indicating that their epitope is located within the overlapping region of fragments I, II and III that consists of amino acids 49-148. Finally, western blotting with monomeric iVHH was performed. VHH were produced with an average concentration of $1.1 \mu \mathrm{g} / \mu \mathrm{l}$. Western blotting using iVHH instead of P-iVHH recognized htt fragments I, II, and III but with less back ground staining.

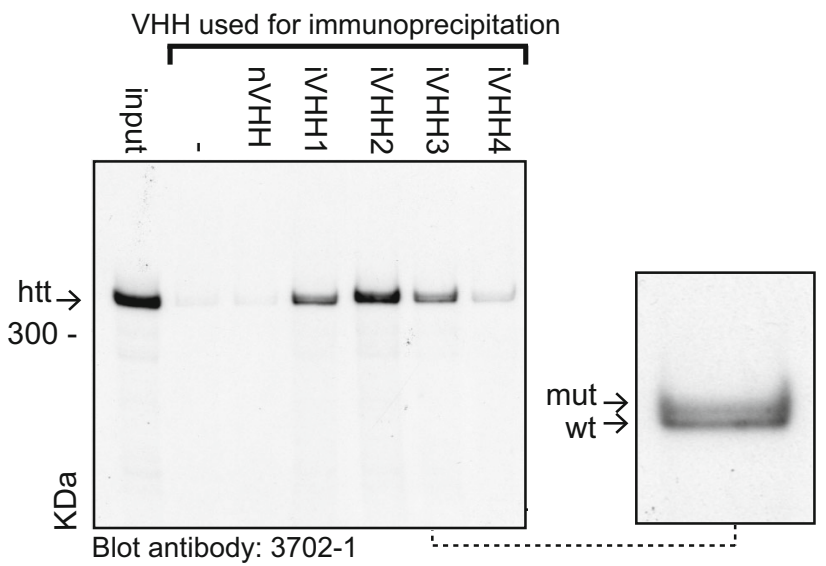

Fig. 4 Immunoprecipitation of endogenous human htt with VHH. Western blot analysis of VHH-htt immunoprecipitation complexes. VHH used for htt immunoprecipitation indicated at top, Input, $10 \mu \mathrm{g}$ of brain lysate; -, No VHH. Arrow indicates full-length htt. $k D a$ Molecular weight (kilodalton). Blot was analysed with 3702-1 anti htt antibody. Right bracket $3 \times$ enlargement of the iVHH3 immunoprecipitation result, showing wild-type (wt) and mutant (mut) huntingtin

iVHH detection of endogenous huntingtin in human HD brain lysates

Next, we analysed if our iVHH were capable of binding endogenous human htt. We performed immunoprecipitation experiments using monomeric iVHH and post-mortem human HD brain lysates. Non-specific binding of htt to the protA-beads without VHH (Fig. 4, -lane) was low. Immunoprecipitation in the presence of iVHH resulted in wild-type and mutant full-length htt band on western blot, while the negative control VHH (nVHH) did not show a full-length htt band (Fig. 4, Online Resource 4). This shows that, in concordance with previous ELISA and western blot results on N-terminal wild-type and mutant htt fragments, our iVHH recognize both wild-type and mutant endogenous full-length htt. Additionally, 
Table 1 Construction of N-terminal Htt fragments

\begin{tabular}{lll}
\hline N-terminal htt fragment & Forward primer & Reverse primer \\
\hline a.a. 1-318 (Q17/Q43) & TATGGCGACCCTGGAAA & GTCGACGAGCAGCACGCCAAGA \\
a.a. 15-378 (Q17/Q43) & CAAGTCCTTCCAGCAGCA & GTCGACGGCTCCGGTCACAACA \\
a.a. $49-415$ (no polyQ) & GCCGCCTCCTCAGCTTC & GTCGACGCCACCAGACTCCTCCTT
\end{tabular}

a.a. amino acid, $Q 17 / Q 43$ polyQ stretch, Underscored SalI-site

immunoprecipitation with iVHH4 was less efficient compared with the other iVHH.

\section{Discussion}

To our knowledge, our study is the first in which VHH have been selected against $\mathrm{N}$-terminal htt protein fragments from an immunized llama phage display library. Monoclonal antibodies are increasingly used as therapeutic agents [13]. Proof of concept for using antibodies in HD has been provided $[3,4]$. Whilst this is promising, there are high demands on any therapeutic or imaging antibody. Because HD is a brain disorder, it is important to cross the blood-brain barrier (BBB). Some VHH were able to cross the BBB [14, 15]. Also, VHH diffusion throughout the brain, and cellular uptake were demonstrated [15]. Although $\mathrm{VHH}$ selection from non-immune phage-VHH libraries is possible [16], selection from immune llama phage-VHH libraries is expected to yield more highaffinity binders because of affinity maturation. However, diversity will be less as affinity maturation selects for the strongest binder. This is reflected in our results. Our iVHH share a high degree of sequence homology. This indicates that the binding epitope of our iVHH is very similar. Our iVHH bind full-length and N-terminal htt in a native and denatured conformation. Binding characteristics for iVHH1-3 were comparable, whereas iVHH4 showed a lower binding efficiency. This is probably due to a single amino acid change in iVHH4 at position 55 in CDR2 where the a-polar alanine was replaced with a polar serine. Because both alanine and serine have side-chains of comparable sizes, it is conceivable that the lower binding efficiency of iVHH4 is due to a shift from an a-polar to polar amino acid. The epitope of all iVHH was mapped to a region between amino acids 49 and 148 downstream of the polyQ repeat, explaining why our iVHH bind both wild-type and mutant htt. In this region, htt contains several domains associated with HD pathogenesis. There is a proline rich region involved in sequestration of other proteins into htt aggregates [17]. Furthermore, proteolytic cleavage between amino acids 104 and 114 was linked with formation of intranuclear aggregates associated with increased toxicity [12]. Binding of our iVHH in this region could alleviate HD pathogenesis. However, it has to be noted that our huntingtin-specific VHH could also block neuroprotective properties of wild-type htt [18]. Additional research is needed to assess the therapeutic properties of our iVHH or their possible applications in HD research. We have demonstrated the feasibility of using VHH for immunoprecipitation, eliminating interference by $\mathrm{IgG}$ bands in the subsequent western blot [19]. Furthermore, since VHH have been used as imaging agent to stain amyloid- $\beta$ deposits in vivo in an Alzheimer disease mouse model [20], our iVHH could be an interesting in vivo imaging tool in HD models to visualize the htt protein.

\section{Materials and methods}

$\mathrm{N}$-terminal htt fragments

A HTT reference sequence with 23 polyQs was used. For PCR primers see Table 1. PCR products were ligated directly into the pGEM-T easy vector (Promega, Madison, WI, USA), digested with NcoI and SalI (Fermentas, St. Leon-Rot, Germany), and ligated into a NcoI/XhoI (Fermentas) pre-digested pIVEX 1.3 vector. Clones were confirmed by sequence analysis. Htt protein fragments were produced with the RTS-100 wheat germ CECF kit (5 PRIME, Gaitersburg, MD, USA) using the ProteoMaster rapid translation system (Roche). To produce the N-terminal htt 1-148 Q46 protein fragment, the HD1955 pCI construct consisting of HTT nucleotides 1-1640 [21] was cloned into a pRP261 vector using the NcoI/SalI restriction sites, and re-cloned into the pET28 vector using the BamHI/SalI restriction sites. The HD1955-pET28 construct was digested with $X h o I$ and self-religated, resulting in the HD828-pET28 construct. Production and purification were performed in BL21 codon+ E.coli cells as described for VHH. To prevent aggregation, dialysis was performed in PBS + $0.5 \%$ Sarkosyl (Sigma-Aldrich).

\section{VHH selection}

Selections were performed as described [16]. The first selection round involved a direct coating of NUNC maxisorp plates (Thermo fisher Scientific, Rochester, NY, USA) 
with 10,5 , or $2.5 \mu \mathrm{g}$ of antigen, or a pre-capturing of $10 \mu \mathrm{g}$ antigen with 2,1 , or $0.5 \mu \mathrm{g}$ of coated $1 \mathrm{H} 6$ antibody (Abnova, Taipei, Taiwan). Phage-VHH (P-VHH) from the first round were produced and purified as described [22] and subjected to a second round of selection with 5, 2.5, or $1 \mu \mathrm{g}$ of directly-coated antigen. Purified P-VHH were stored at $-20{ }^{\circ} \mathrm{C}$ in PBS containing $10 \%$ glycerol. TG1 E. coli cells were infected with phage-VHH from the second selection round and plated on LB/Agar containing ampicillin. Ninetyfour randomly selected clones were tested as described [10]. As secondary antibody for the screening ELISA, we used HRP conjugated mouse anti M13 (GE Healthcare, Buckinghamshire, UK). VHH DNA was purified using the Nucleospin Plasmid purification kit (Macherey-Nagel, Duren, Germany) according to manufacturer's instructions. DNA was sequenced using primer M13REV (CAGGAAA CAGCTATGAC). DNA to protein conversion: http://www. bioinformatics.picr.man.ac.uk/research/software/tools/sequenceconverter.html. VHH sequence alignment: http://www. ebi.ac.uk/Tools/msa/clustalW2.

\section{VHH production}

PCR was performed on M13 plasmid using primers iVHHFW (CGGAATTCCTTTAGTTGTTCCA), and iVHH-Rev (CACATCATCATCACCATCACG), or nVHH-FW (CGCT GGATTGTTATTACTCGC) and nVHH-Rev (CCTCA GAACCCAAGACCA). PCR fragments were cloned into pUR5850 [23] by SfiI and BstEII (Fermentas) digestion and ligation. Clones were verified by sequence analysis and transferred into Neb5 E. coli for production. $\mathrm{VHH}$ were purified using the His6-tag with TALON metal affinity resin (Clontech, Mountain View CA, USA) according to the manufacturer's instructions using $50 \mathrm{mM}$ NaPO4, $0.3 \mathrm{M}$ $\mathrm{NaCl}, \mathrm{pH} 7$ as wash buffer. Wash buffer with $150 \mathrm{mM}$ imidazole was used as elution buffer. Pooled eluates were dialyzed against PBS using Cellu-Sep dialyze-tube MWCO 3500 (Interchim, MontluÇon, France). VHH production was checked on Coomassie staining (PageBlue, Thermo-Scientific) and western blotting against the VSV-tag. VHH was quantified with bicinchoninic assay (BCA, Thermo-Scientific). VHH were stored in $5 \%$ glycerol at $-20{ }^{\circ} \mathrm{C}$.

\section{ELISA}

A 96-well NUNC maxisorp plate was coated with $0.1 \mu \mathrm{g} /$ well of antigen. Staining was performed using $\mathrm{P}-\mathrm{VHH}$ diluted 1:20, followed by HRP conjugated mouse anti M13 (Millipore Billerica, MA, USA). ELISA signal was visualized with $o$-Phenylenediamine (OPD, Sigma-Aldrich). Optical density at $\lambda=490 \mathrm{~nm}$ was measured using a plate reader (Biotek, Winooski, USA). The huntingtin-specific antibody MAB5492 (Millipore) was used as positive control.

Western blot

N-terminal htt fragments were run on a $10 \%$ SDS-PAGE gel and proteins were blotted onto nitrocellulose membrane (\#170-4159, Bio-Rad, Hercules, CA, USA). Blots were blocked with $4 \%$ non-fat milk (Nutricia, Schiphol, The Netherlands) in TBST. Primary antibodies: 3702-1 (Epitomics, Burlinggame, CA, USA), MAB5492 (Millipore), 1 H6 (Abnova, Taipei city, Taiwan), P-VHH or $20 \mathrm{ng} / \mu \mathrm{l}$ VHH. Blots probed with VHH were subsequently incubated with mouse anti VSV (Cell signalling). Secondary antibodies: HRP conjugated goat anti-mouse, goat antirabbit (Santa-Cruz), or mouse anti-M13 (Millipore). Bands were detected with Amersham ECL (\#RPN 2132, GE healthcare) and Hyperfilm ECL (\#28906837, GE healthcare).

Immunoprecipitation assay

Human post-mortem brain tissue from the middle temporal gyrus of a 67 year old female HD subject (CAG1: 15, CAG2: 42) was obtained with the families full consent and the ethical approval of the various institutional Ethics Committees. Post-mortem delay was $9 \mathrm{~h}$. VHH $(15 \mu \mathrm{g})$ was bound to $10 \mu \mathrm{l}$ bed volume of protein A sepharose beads (GE Healthcare), and incubated for $90 \mathrm{~min}$ with $100 \mu \mathrm{g}$ of post-mortem human HD brain tissue lysate in PBS. Western blot analysis of the VHH-htt complexes was performed as described above, where SDS-PAGE was performed according to [24].

Acknowledgments We thank Richard L. M. Faull and the Neurological Foundation of New Zealand Human Brain Bank for the HD post-mortem brain tissue. We thank Dr. Michael Hayden for the HD1955 (CAG44)-pCI construct. We thank Fausta Bankauskaite for technical assistance. This study was supported by a grant (WAR30808) from the Prinses Beatrix Spierfonds, and the Centre for Biomedical Genetics of the Netherlands.

Conflict of interest The authors declare no competing financial interests.

Open Access This article is distributed under the terms of the Creative Commons Attribution License which permits any use, distribution, and reproduction in any medium, provided the original author(s) and the source are credited.

\section{References}

1. (1993) A novel gene containing a trinucleotide repeat that is expanded and unstable on Huntington's disease chromosomes. 
The Huntington's Disease Collaborative Research Group. Cell 72(6): 971-983

2. Jacobsen JC, Gregory GC, Woda JM et al (2011) HD CAGcorrelated gene expression changes support a simple dominant gain of function. Hum Mol Genet 20(14):2846-2860. doi:10. 1093/hmg/ddr195

3. Wolfgang WJ, Miller TW, Webster JM et al (2005) Suppression of Huntington's disease pathology in Drosophila by human single-chain Fv antibodies. Proc Natl Acad Sci USA 102(32):11563-11568. doi:10.1073/pnas.0505321102

4. Snyder-Keller A, McLear JA, Hathorn T, Messer A (2010) Early or late-stage anti-N-terminal Huntingtin intrabody gene therapy reduces pathological features in B6.HDR6/1 mice. J Neuropathol Exp Neurol 69(10):1078-1085. doi:10.1097/NEN.0b013e3181f530ec

5. Hamers-Casterman C, Atarhouch T, Muyldermans S et al (1993) Naturally occurring antibodies devoid of light chains. Nature 363(6428):446-448. doi:10.1038/363446a0

6. van der Linden RH, Frenken LG, de Geus B et al (1999) Comparison of physical chemical properties of llama VHH antibody fragments and mouse monoclonal antibodies. Biochim Biophys Acta 1431(1):37-46

7. Arbabi Ghahroudi M, Desmyter A, Wyns L et al (1997) Selection and identification of single domain antibody fragments from camel heavy-chain antibodies. FEBS Lett 414(3):521-526

8. Verheesen P, de Kluijver A, van Koningsbruggen S et al (2006) Prevention of oculopharyngeal muscular dystrophy-associated aggregation of nuclear polyA-binding protein with a singledomain intracellular antibody. Hum Mol Genet 15(1):105-111. doi: $10.1093 / \mathrm{hmg} / \mathrm{ddi} 432$

9. Chartier A, Raz V, Sterrenburg E et al (2009) Prevention of oculopharyngeal muscular dystrophy by muscular expression of Llama single-chain intrabodies in vivo. Hum Mol Genet 18(10):1849-1859. doi:10.1093/hmg/ddp101

10. Pepers BA, Schut MH, Vossen RH et al (2009) Cost-effective HRMA pre-sequence typing of clone libraries; application to phage display selection. BMC Biotechnol 9:50. doi:10.1186/ 1472-6750-9-50

11. Liu B, Huang L, Sihlbom C et al (2002) Towards proteome-wide production of monoclonal antibody by phage display. J Mol Biol 315(5):1063-1073. doi:10.1006/jmbi.2001.5276

12. Lunkes A, Lindenberg KS, Ben-Haiem L et al (2002) Proteases acting on mutant huntingtin generate cleaved products that differentially build up cytoplasmic and nuclear inclusions. Mol Cell 10(2):259-269

13. Leavy O (2010) Therapeutic antibodies: past, present and future. Nat Rev Immunol 10(5):297. doi:10.1038/nri2763
14. Rutgers KS, Nabuurs RJ, van den Berg SA et al (2011) Transmigration of beta amyloid specific heavy chain antibody fragments across the in vitro blood-brain barrier. Neuroscience 190:37-42. doi:10.1016/j.neuroscience.2011.05.076

15. Li T, Bourgeois JP, Celli S et al (2012) Cell-penetrating antiGFAP VHH and corresponding fluorescent fusion protein VHHGFP spontaneously cross the blood-brain barrier and specifically recognize astrocytes: application to brain imaging. FASEB J 26(10):3969-3979. doi:10.1096/fj.11-201384

16. Verheesen P, Roussis A, de Haard HJ et al (2006) Reliable and controllable antibody fragment selections from Camelid nonimmune libraries for target validation. Biochim Biophys Acta 1764(8):1307-1319. doi:10.1016/j.bbapap.2006.05.011

17. Qin ZH, Wang Y, Sapp E et al (2004) Huntingtin bodies sequester vesicle-associated proteins by a polyproline-dependent interaction. J Neurosci 24(1):269-281. doi:10.1523/JNEUR OSCI.1409-03.2004

18. Dragatsis I, Levine MS, Zeitlin S (2000) Inactivation of Hdh in the brain and testis results in progressive neurodegeneration and sterility in mice. Nat Genet 26(3):300-306. doi:10.1038/81593

19. Landles C, Sathasivam K, Weiss A et al (2010) Proteolysis of mutant huntingtin produces an exon 1 fragment that accumulates as an aggregated protein in neuronal nuclei in Huntington disease. J Biol Chem 285(12):8808-8823. doi:10.1074/jbc.M109.075028

20. Nabuurs RJ, Rutgers KS, Welling MM et al (2012) In vivo detection of amyloid-beta deposits using heavy chain antibody fragments in a transgenic mouse model for Alzheimer's disease. PLoS ONE 7(6):e38284. doi:10.1371/journal.pone.0038284

21. Martindale D, Hackam A, Wieczorek A et al (1998) Length of huntingtin and its polyglutamine tract influences localization and frequency of intracellular aggregates. Nat Genet 18(2):150-154. doi:10.1038/ng0298-150

22. Marks JD, Hoogenboom HR, Bonnert TP et al (1991) By-passing immunization. Human antibodies from V-gene libraries displayed on phage. J Mol Biol 222(3):581-597

23. Verheesen P, ten Haaft MR, Lindner N et al (2003) Beneficial properties of single-domain antibody fragments for application in immunoaffinity purification and immuno-perfusion chromatography. Biochim Biophys Acta 1624(1-3):21-28

24. Hu J, Matsui M, Gagnon KT et al (2009) Allele-specific silencing of mutant huntingtin and ataxin-3 genes by targeting expanded CAG repeats in mRNAs. Nat Biotechnol 27(5):478-484. doi:10. 1038/nbt.1539

25. Kabat E, Wu TT, Perry H et al. (1991) United States Public Health Services Publication No. 91-3242 (National Institutes of Health, Bethesda, MD) 